\title{
Tool manufacture by naive juvenile crows
}

\section{The use of twigs by these birds to coax out hidden food seems to be an instinctive skill.}

$\mathrm{N}$ ew Caledonian crows (Corvus moneduloides) are the most prolific avian toolusers $^{1,2}$. Regional variation in the shape of their tools may be the result of cumulative cultural evolution ${ }^{3}$ - a phenomenon considered to be a hallmark of human culture ${ }^{4}$. Here we show that hand-raised juvenile New Caledonian crows spontaneously manufacture and use tools, without any contact with adults of their species or any prior demonstration by humans. Our finding is a crucial step towards producing informed models of cultural transmission in this species, and in animals in general.

Using four juveniles (three males, one female) bred in our captive colony in 2004, we tested whether New Caledonian crows have inherited characteristics that support tool-making and use. We hand-raised chicks in artificial nests and subsequently transferred them to enriched aviaries that contained twigs of assorted shapes and sizes, and food items hidden in holes and crevices. None of the subjects was ever allowed to observe an adult crow. Two of them, a male and a female, were housed together and were given regular demonstrations by their human foster parents of how to use twig tools to retrieve food. The other two were housed individually and never witnessed tool use; one of them, named Corbeau, never saw objects being handled that he could have used as a tool.

All four crows developed the ability to use twig tools (Fig. 1a). (For movies showing the events described here, see supplementary information.) Although the tutored crows paid close attention to demonstrations, we observed no qualitative difference between them and the untutored birds in their tooloriented behaviour. We first observed successful food retrieval from a crevice by the tutored birds when they were 68 and 72 days old, and by the untutored birds at 63 and 79 days old. All juveniles continue to use twig tools to probe holes or crevices whenever the opportunity is provided.

We also tested the juveniles' response to leaves from trees of the genus Pandanus, similar to those from which wild individuals make tools that vary regionally in shape and complexity ${ }^{3}$. We mounted the leaves on wooden frames so that the birds could access them roughly as they would in the wild. On the first day that he was presented with Pandanus, Corbeau (then aged 99 days) produced a straight tool, 13 centimetres long, from one side of the leaf by using a swift 'cut-tear-cut' action. Immediately after producing the tool (Fig. 1b), Corbeau carried it to a crevice where food was often hidden and

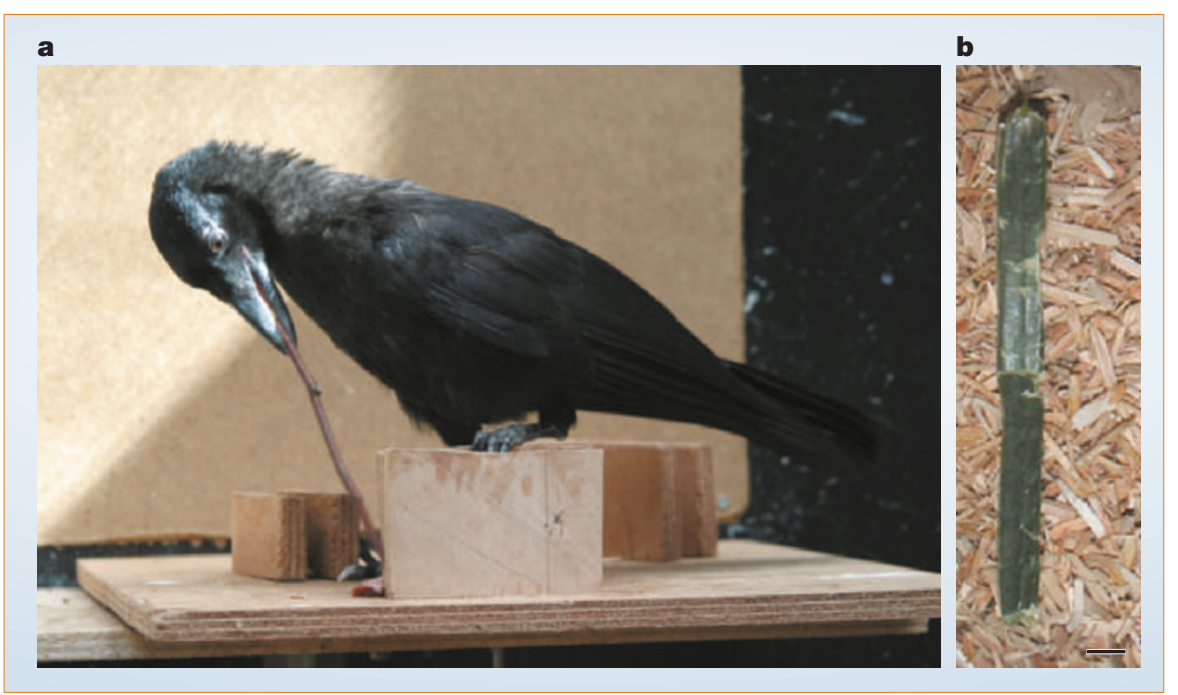

Figure 1 Tool use by a naive New Caledonian crow. a, A hand-raised juvenile uses a twig to retrieve meat from an artificial crevice. This individual has never witnessed tool use by a conspecific or by its human foster parents. b. Close-up of a tool made from a Pandanus leaf (provided by the Royal Botanic Gardens, Kew, London) by the same bird (see movie in supplementary information); scale bar, $1 \mathrm{~cm}$. This work was carried out in accordance with the University of Oxford's procedures for local ethical review.

used it as a probe, a sequence that he has since repeated several times, successfully retrieving food.

All four crows attacked the leaves, cutting and tearing them into a variety of different shapes: only some of these would have been suitable as tools and none resembled the distinct 'stepped-cut' Pandanus tools fashioned by adults in the wild ${ }^{3}$. So far, we have observed only Corbeau using leaf pieces to retrieve food.

These results show that the ability of this species to manufacture and use tools is at least partly inherited and not dependent on social input.Spontaneous tool use has been recorded in a range of primate species ${ }^{5,6}$ and in the woodpecker finch (Cactospiza pallida) ${ }^{7}$, the only other bird known to use stick tools regularly in the wild. However, to our knowledge, ours is the first demonstration of spontaneous tool manufacture in a naive juvenile vertebrate - previous descriptions of manufacture concern groups of primates containing adults with prior experience of tool use $\mathrm{e}^{8,9}$.

In the light of our findings, it is possible that the high level of skill observed in wild adult crows is not socially acquired. Social input, however, may be important in transmitting specific techniques ${ }^{10}$ and tool shapes ${ }^{3}$. This idea is supported by the close attention our juveniles paid to demonstrations of tool use by their human foster parents.

The fact that an inherited predisposition can account for a complex behaviour such as tool manufacture highlights the need for controlled investigation into behavioural ontogeny in other species that seemingly show culturally transmitted behaviour. The
New Caledonian crow could be a valuable model for investigating interactions between inherited traits and individual ${ }^{11}$ and social ${ }^{3}$ learning during the development of tool technology — an issue central to the understanding of the emergence of human culture.

Ben Kenward, Alex A. S. Weir,

Christian Rutz, Alex Kacelnik

Department of Zoology, University of Oxford,

South Parks Road, Oxford OX1 3PS, UK

e-mail: alex.kacelnik@zoology.ox.ac.uk

1. Kenward, B., Rutz, C., Weir, A. A. S., Chappell, J. \& Kacelnik, A. Ibis 146, 652-660 (2004)

2. Hunt, G. R. Nature 379, 249-251 (1996)

3. Hunt, G. R. \& Gray, R. D. Proc. R. Soc. Lond. B 270, 867-874 (2003)

4. Tomasello, M. Annu. Rev. Anthropol. 28, 509-529 (1999).

5. Westergaard, G. C. Percept. Mot. Skills 68, 558 (1989).

6. Spaulding, B. \& Hauser, M. Anim. Behav. (in the press).

7. Tebbich, S., Taborsky, M., Fessl, B. \& Blomqvist, D. Proc. R. Soc. Lond. B 268, 2189-2193 (2001).

8. Westergaard, G. C. \& Fragaszy, D. M. J. Comp. Psychol. 101, 159-168 (1987)

9. Celli, M. L., Hirata, S. \& Tomonaga, M. Int. J. Primatol. 25, 1267-1281 (2004).

10. Hunt, G. R. \& Gray, R. D. Proc. R. Soc. Lond. B (suppl.) 271, 88-90 (2004).

11. Weir, A. A. S., Chappell, J. \& Kacelnik, A. Science 297, 981 (2002) Supplementary information accompanies this communication on Nature's website.

Competing financial interests: declared none.

brief communications arising online

www.nature.com/bca

Arbuscular mycorrhizal fungi: Hyphal fusion and multigenomic structure

J. D. Bever, M. Wang (doi:10.1038/nature03294)

Reply: T. E. Pawlowska, J. W. Taylor

(doi:10.1038/nature03295) 